\title{
Interplay between shell structure and trap deformation in dipolar Fermi gases
}

\author{
J. Bengtsson ๑," G. Eriksson ๑, J. Josefi, J. C. Cremon, and S. M. Reimann $\odot$ \\ Mathematical Physics and NanoLund, Lund University, Box 118, 22100 Lund, Sweden
}

(Received 5 April 2020; accepted 21 September 2020; published 4 November 2020)

\begin{abstract}
Finite fermion systems are known to exhibit shell structure in the weakly interacting regime, as is well known from atoms, nuclei, metallic clusters, or even quantum dots in two dimensions. All these systems have in common that the particle interactions between electrons or nucleons are spatially isotropic. Dipolar quantum systems as they have been realized with ultracold gases, however, are governed by an intrinsic anisotropy of the two-body interaction that depends on the orientation of the dipoles relative to each other. Here we investigate how this interaction anisotropy modifies the shell structure in a weakly interacting two-dimensional anisotropic harmonic trap. Going beyond Hartree-Fock analysis by applying the so-called importance-truncated configuration-interaction (CI) method as well as quadratic CI with single and double substitutions, we show how the magnetostriction in the system may be counteracted upon by a deformation of the confinement.
\end{abstract}

DOI: 10.1103/PhysRevA.102.053302

\section{INTRODUCTION}

Atomic alkali-metal clusters are one of the first experimentally realized man-made artificial quantum systems with mass spectra revealing pronounced electronic shells $[1,2]$ analogous to the closed shells in atomic noble gases, or the "magic numbers" of increased stability well known from nuclear structure [3]. Another example is semiconductor quantum dots where the low-dimensional electron gas in a heterostructure can be electrostatically confined to small electron puddles exhibiting shell structure [4]. A third, yet very different, category of finite quantum systems is offered by the advances with trapped cold atomic quantum gases (see, for example, the reviews and books in Refs. [5-8]). These systems offer appealing possibilities to realize and simulate a wide range of quantum many-body phenomena [9], for bosonic as well as for fermionic atoms. In contrast to clusters or nanostructures, there is a very high degree of tunability: Not only can one design the shape and dimensionality of the confinement, but one can also modify both strength and shape of the particle interactions. It also became possible to reach the few-body limit [10-13], even down to single-atom control in experiments with fermionic ${ }^{6} \mathrm{Li}[13]$.

Initially, studies of ultracold and dilute atomic quantum gases mainly considered isotropic short-range interactions $[6,7]$. Atoms such as chromium have a large magnetic dipole moment, but usually the isotropic van der Waals interactions dominate. However, tuning these interactions by Feshbach resonances may enhance the relative strength of the dipoledipole interactions $[14,15]$, bringing their long-range nature

\footnotetext{
*jakob.bengtsson@matfys.lth.se
}

Published by the American Physical Society under the terms of the Creative Commons Attribution 4.0 International license. Further distribution of this work must maintain attribution to the author(s) and the published article's title, journal citation, and DOI. Funded by Bibsam. and spatial anisotropy into play. After reaching the milestone of realizing a dipolar Bose-Einstein condensate [16,17], many other experiments followed [18,19], also for dysprosium [20-22] and erbium [23] and polar molecules [24-30]. Also dipolar Fermi gases of dysprosium [31], fermionic erbium [32], and a chromium dipolar Fermi sea [33] were reported. (For early reviews on dipolar gases, see, e.g., Refs. [19,34,35]). More recently, with erbium atoms it also became possible to realize two-component Fermi gases with strong and tunable interactions [36].

On the theory side, trapped dipolar Fermi gases have been studied extensively within variational approaches such as Hartree-Fock and beyond (see, for example, Refs. [37-48]). Reference [37] showed that the Fock exchange term leads to a deformation in momentum space. The Hartree term alone resulted in a deformation in position space [49], leading to an instability of spherically trapped gases, but stabilizing prolate or oblate ones. The spheroidal distortion of the Fermi surface predicted in Ref. [37] was then also observed experimentally (see Ref. [50]).

In a most simple description, shell structure occurs as a consequence of the distribution of single-particle energies that are associated with the approximation by an effective meanfield potential. The underlying principle is similar in cases where the interactions are relatively weak, in other words, when correlation effects are not too strong. A high degree of symmetry of the system initially leads to degeneracies and a "bunchiness" of levels in the mean-field single-particle spectrum. When the fermion number is such that a bunch of energy levels can be fully occupied and there is a gap at the Fermi surface, i.e., when a "shell" is filled, the system is particularly stable. In an open-shell scenario, however, where the particle number is insufficient to fill the shell, the system will undergo a breaking of symmetry to reach stability at different level fillings by lowering the degree of degeneracy. In self-bound fermion systems such as nuclei or metallic clusters where interactions are mainly spatially isotropic, this leads to the well-known Jahn-Teller shape deformations [2,3]. 
In dipolar quantum gases, in contrast, the dipole-dipole twobody interaction itself is spatially anisotropic and depends on the orientation of the dipoles relative to each other. In this case an anisotropy of the effective mean field may originate rather from the intrinsic structure of the two-body force than from the trap, confining the gas by a potential with an externally determined shape.

We here thus pose the question, whether shell effects may occur for fermionic dipolar gases where (electric or magnetic) dipole moments may be aligned by a corresponding external field, and how the shell structure modifies upon a tilt of the dipole direction. In three dimensions, the unavoidable head-to-tail attraction would prevent the realization of a purely repulsive system. We thus here restrict our analysis to a quasi-two-dimensional harmonic trap, where the azimuthal symmetry yields a strong shell structure despite the reduced dimensionality [4], but where for moderate tilt angles the otherwise dominant head-to-tail attraction can be avoided $[25,26]$.

Early studies of both energy- and density-shell structures in trapped Fermi gases were reported in Refs. [51-53], followed by a time-dependent Hartree-Fock analysis of shell structure in three dimensions in Ref. [54]. Importantly, in the latter work it was shown that quadrupole and breathing modes are less affected in the cases of closed spherical shells. References $[47,48,55]$ more recently investigated the effect of the dipolar anisotropy on the time-of-flight expansion and on the angular dependence of the Fermi surface deformation of the ground state in relation to the trap anisotropy, and found that the Fermi surface deforms maximally when the dipoles are tilted along the less confined trap direction. Here, we analyze the interplay of this effect with the otherwise predominant shell structure in the weakly interacting regime.

The enhancement of interaction effects in two-dimensional dipolar Fermi gases and the importance to go beyond HartreeFock analysis was discussed in Refs. [56-58]. We here apply the so-called importance-truncated configuration-interaction (CI) method [59] as well as quadratic CI [60] with single and double substitutions, allowing us to go beyond Hartree-Fock analysis.

The paper is organized as follows: In Sec. II we describe the setup and the effective dipole-dipole interaction in a quasitwo-dimensional confinement, and describe in Sec. III the methodology. In Sec. IV we discuss the low-lying energy eigenstates as a function of the dipolar anisotropy for a deformed confinement, mapping out the interplay between the trap confinement and the magnetostriction associated with the dipolar two-body interaction. We finally analyze the second differences in the ground-state energies, which is a measure for the strength of the shell structure in the symmetric, anisotropic, and symmetry-restored cases, in Sec. V, and conclude in Sec.VI.

\section{DIPOLES IN A QUASI-TWO-DIMENSIONAL HARMONIC TRAP}

Let us now consider $N$ spin-polarized fermions in a trapping potential $V^{\text {trap }}(\boldsymbol{r})=V_{z}^{\text {trap }}(z) V_{\perp}^{\text {trap }}\left(\boldsymbol{r}_{\perp}\right)$, where $\boldsymbol{r}=(x, y, z)$ and $\boldsymbol{r}_{\perp}=(x, y)$. Here, $V_{z}^{\text {trap }}$ is a tightly confined harmonic oscillator (with angular frequency $\omega_{z}$ ), such that all particles may be described by the (single-particle) Gaussian ground state in the $z$ direction, and

$$
V_{\perp}^{\text {trap }}\left(\boldsymbol{r}_{\perp}\right)=\frac{1}{2} M \omega_{\perp}^{2}\left(\alpha x^{2}+\alpha^{-1} y^{2}\right)
$$

is a parity-conserving potential, where $M$ is the particle mass, $\omega_{\perp}=0.01 \omega_{z}$, and $\alpha=1.15$. The constant $\alpha$ determines the anisotropy of the trap in the $x y$ plane. The dipole moment vectors $\boldsymbol{d}$ associated with the fermions are aligned by an external field. The interaction potential $V^{\text {int }}$ between two particles, with identical vectors $\boldsymbol{d}$, respectively at positions $\boldsymbol{r}$ and $\boldsymbol{r}^{\prime}$, reads

$$
V^{\mathrm{int}}(\tilde{\boldsymbol{r}})=D^{2} \frac{1-3 \cos ^{2} \theta_{\tilde{\boldsymbol{r}} \boldsymbol{d}}}{|\tilde{\boldsymbol{r}}|^{3}},
$$

where $D^{2}=0.8^{2} \hbar \omega_{\perp} l_{\perp}^{3}$ is the coupling (or interaction) strength, $l_{\perp}=\sqrt{\hbar /\left(M \omega_{\perp}\right)}$ is the oscillator length in the $x y$ plane, $\tilde{\boldsymbol{r}}=\boldsymbol{r}-\boldsymbol{r}^{\prime}$ is the relative position, and $\theta_{\tilde{\boldsymbol{r}} \boldsymbol{d}}$ is the angle between $\tilde{\boldsymbol{r}}$ and $\boldsymbol{d}$. The tight confinement in the $z$ direction effectively reduces the three-dimensional potential, $V^{\text {int }}$, to a quasi-two-dimensional one, $V_{\perp}^{\text {int }}$. As in Ref. [61] we assume that $\boldsymbol{d}$ is oriented in the $x z$ plane by an angle $\Theta$ to the $x$ axis, and obtain

$$
\begin{aligned}
V_{\perp}^{\text {int }}\left(\tilde{\boldsymbol{r}}_{\perp}\right)= & \frac{D^{2} e^{\tilde{\xi}}}{2 \sqrt{2 \pi} l_{z}^{3}}\left\{(2+4 \tilde{\xi}) K_{0}(\tilde{\xi})-4 \tilde{\xi} K_{1}(\tilde{\xi})\right. \\
& +\cos ^{2} \Theta\left[-(3+4 \tilde{\xi}) K_{0}(\tilde{\xi})+(1+4 \tilde{\xi}) K_{1}(\tilde{\xi})\right] \\
& \left.+2 \cos ^{2} \Theta \cos ^{2} \tilde{\phi}\left[-2 \tilde{\xi} K_{0}(\tilde{\xi})+(2 \tilde{\xi}-1) K_{1}(\tilde{\xi})\right]\right\}
\end{aligned}
$$

where $\tilde{\xi}=\tilde{\boldsymbol{r}}_{\perp}^{2} /\left(4 l_{z}^{2}\right)=\left(\tilde{x}^{2}+\tilde{y}^{2}\right) /\left(4 l_{z}^{2}\right), l_{z}=\sqrt{\hbar /\left(M \omega_{z}\right)}$ is the oscillator length in the $z$ direction, and $\tilde{\phi}=\arctan (\tilde{y} / \tilde{x})$, and where $K_{0}$ and $K_{1}$ are modified Bessel functions of the second kind. The effective dipole-dipole interaction is spatially isotropic when $\Theta=90^{\circ}$, but for smaller angles $\Theta$, it has a pronounced spatial anisotropy. In particular, for $\Theta<90^{\circ}$ a region is formed where $V_{\perp}^{\text {int }}\left(\tilde{\boldsymbol{r}}_{\perp}\right)<0$. For angles in the interval $\arccos (1 / \sqrt{3})<\Theta<90[$ where $\arccos (1 / \sqrt{3}) \approx$ $54.7^{\circ}$ is the so-called magic angle] the dipole-dipole interaction is attractive only for $\tilde{\boldsymbol{r}}_{\perp}$ within a bounded region in $\mathbb{R}^{2}$, whereas for $\Theta<\arccos (1 / \sqrt{3})$ the corresponding region is unbounded. The interaction potential is sketched in Fig. 1 for $\Theta=50^{\circ}$. (The inset shows the shell degeneracies in a two-dimensional isotropic harmonic trap). The effective Hamiltonian of the system here thus reads

$$
\hat{H}=\sum_{i=1}^{N}\left[\frac{\hat{\boldsymbol{p}}_{\perp, i}^{2}}{2 M}+V_{\perp}^{\mathrm{trap}}\left(\hat{\boldsymbol{r}}_{\perp, i}\right)\right]+\sum_{i>j}^{N} V_{\perp}^{\mathrm{int}}\left(\hat{\boldsymbol{r}}_{\perp, i}-\hat{\boldsymbol{r}}_{\perp, j}\right),
$$

where $V_{\perp}^{\text {trap }}$ was given in Eq. (1) above, $V_{\perp}^{\text {int }}$ in Eq. (3), and $\hat{\boldsymbol{p}}_{\perp, i}=\left(\hat{p}_{i, x}, \hat{p}_{i, y}\right)$ and $\hat{\boldsymbol{r}}_{\perp, i}=\left(\hat{x}_{i}, \hat{y}_{i}\right)$ are respectively the momentum and the position operator of the $i$ th particle in the $x y$ plane. For the considered system, we observe that parity is preserved. The individual many-body eigenstate to $\hat{H}$ has thus either even or odd parity.

\section{GOING BEYOND HARTREE-FOCK}

We now search for the low-energy eigenstates to $\hat{H}$ in Eq. (4) for $N=1,2, \ldots, N_{\max }$ fermions and for different 




FIG. 1. Effective interaction potential $V_{\perp}^{\text {int }}$, given by Eq. (3), for interaction strength $D^{2}=0.8^{2} \hbar \omega_{\perp} l_{\perp}^{3}$, oscillator length $l_{\perp}=10 l_{z}$, and a dipolar angle of $\Theta=50^{\circ}$. The inset to the upper right shows a schematic picture of the quasi-two-dimensional harmonic trapping potential $(\alpha=1)$ and its single-particle states in the first four shells, where the white circles schematically represent the single orbitals with shell degeneracies $d_{N_{0}}=N_{0}+1, N_{0}=0,1,2, \ldots$, in two dimensions.

dipolar orientations $\Theta$. Depending on $N_{\max }$, two different numerical methods are employed for $N>1$ : the so-called importance-truncated configuration interaction (ITCI) [59] and the quadratic configuration interaction with single and double substitutions (QCI-SD) [60]. (For $N=1$, the solution is trivially known from the single-particle oscillator ground state). In all methods, the same primitive one-body basis

$$
\varphi_{m, j}\left(r_{\perp}, \phi\right)=e^{i m \phi} B_{j}^{(k)}\left(r_{\perp}\right)
$$

is used at the most fundamental level, where $\left(r_{\perp}, \phi\right)$ are the polar coordinates, $m$ is an integer, and $B_{j}^{(k)}$ is a $k$ th-order $B$-spline. The $B$-splines are piecewise polynomials defined by their order $k$ and by their so-called knot-point sequence $\tau_{j} \leqslant \tau_{j+1}$ (see, e.g., Ref. [62]),

$$
\begin{aligned}
B_{j}^{(1)}\left(r_{\perp}\right)= & \begin{cases}1 & \text { if } \tau_{j} \leqslant r_{\perp}<\tau_{j+1} \\
0 & \text { otherwise, }\end{cases} \\
B_{j}^{(k)}\left(r_{\perp}\right)= & \frac{r_{\perp}-\tau_{j}}{\tau_{j+k-1}-\tau_{j}} B_{j}^{(k-1)}\left(r_{\perp}\right) \\
& +\frac{\tau_{j+k}-r_{\perp}}{\tau_{j+k}-\tau_{j+1}} B_{j+1}^{(k-1)}\left(r_{\perp}\right) .
\end{aligned}
$$

In total, we use 702 single-particle basis states. The angular part of $\varphi$ is limited to $m \in[-13,13]$ and $26 B$-splines are used to sample the radial part. We chose fifth-order $B$-splines, i.e., $k=5$, defined by a linear distribution of knot points with $\Delta \tau=0.15 l_{\perp}$ in an inner region, $0 \leqslant r_{\perp} / l_{\perp} \leqslant 3$, and an exponentially increasing distance between the knot points in an outer region, $3 \leqslant r_{\perp} / l_{\perp} \leqslant 5$. The last knot point, located at $r_{\perp}=5 l_{\perp}$, sets the radius of our computational box. The computational basis is carefully chosen to ensure an adequate description of systems within a rather wide range of dipolar orientations $\left(45^{\circ} \leqslant \Theta \leqslant 90^{\circ}\right)$ and particle numbers $(N \leqslant 16)$, while keeping the basis size down and the computational time tractable. We note that the systems with predominantly repulsive dipole-dipole interactions, i.e., where $\Theta \approx 90^{\circ}$, will effectively determine the required computational box radius. Naturally, the lower limit on the box radius grows with the particle number $N$ of the system (as well as with a possible increase in the interaction strength $D^{2}$ ). A decrease in $\Theta$, on the other hand, makes it increasingly important (particularly for $\Theta$ below the magic angle) to numerically resolve the effects of the attractive dipole-dipole interaction region. Consequently, the systems with $\Theta=45^{\circ}$, i.e., the lowest $\Theta$ here considered, effectively sets the upper limit on $\Delta \tau$.

For a systematic comparison of systems with only a handful of fermions (here for $N \leqslant 4$ ), the eigenenergies and eigenstates of $\hat{H}$ are here retrieved using the method of ITCI. We divide the full many-body Hilbert space $\mathcal{H}$ into a model (or reference) subspace $\mathcal{H}_{M}$ and the orthogonal complementary $\mathcal{H}_{C}$. To reduce the computational workload, the diagonalization of $\hat{H}$ is performed within $\mathcal{H}_{M}$ alone. ITCI is thus variational, with the many-body eigenenergies $E$ obtained with $\mathcal{H}_{M}$ replacing (as estimates of) the eigenenergies using the full $\mathcal{H}$. Furthermore, we explore the possibility to tailor $\mathcal{H}_{M}$ for one specific many-body solution at a time, with the purpose of keeping the dimensionality of $\mathcal{H}_{M}$ (for each retrieved eigenenergy) to a minimum. For the singly targeted many-body solution, the validity of the model space is estimated using perturbation theory. In particular, we expand the desired many-body solution (retrieved with $\mathcal{H}_{M}$ ) to $\mathcal{H}_{C}$ following the prescription of multiconfigurational first-order perturbation theory (here based on an Epstein-Nesbet-like [63,64] partitioning, as discussed in, e.g., Ref. [59]). The corresponding second-order energy correction $\delta E^{(2)}$ to the many-body eigenenergy $E$ serves as an error estimate of $\mathcal{H}_{M}$. (The first-order correction $\delta E^{(1)}=0$ by construction). Of course, the perturbative correction to the many-body solution also identifies the most important basis states belonging to $\mathcal{H}_{C}$. This information promotes an iterative construction of $\mathcal{H}_{M}$, starting from an initial guess spanned by only a few many-body basis states, and transferring the most important states from $\mathcal{H}_{C}$ to $\mathcal{H}_{M}$ in each iteration. Here, the many-body basis is given by Fock states, constructed from orthogonal one-body functions. However, since the $B$-splines are nonorthogonal, we chose (for simplicity) to diagonalize the single-particle (isotropic) harmonic oscillator Hamiltonian in the $\varphi$ basis [given by Eq. (5)] and use these solutions when constructing the many-body basis. A subset of the hereby acquired Fock states will furthermore span $\mathcal{H}_{M}$ and its complementary will span $\mathcal{H}_{C}$. In each iteration, the latter Fock states are assessed based on the sizes of their individual overlaps with the latest computed desired (first-order corrected) many-body solution. Next, we transfer all Fock states from $\mathcal{H}_{C}$ to $\mathcal{H}_{M}$ with large enough overlap magnitudes (here we chose a threshold of 5\% compared to the largest sized overlap identified among all Fock states in $\mathcal{H}_{C}$ ). With this approach, $\mathcal{H}_{M}$ systematically approaches $\mathcal{H}$. In principle, the eigenenergies retrieved with ITCI thus converge to that obtained with full configuration interaction (using $\mathcal{H}$ ). In practice, however, we stop the incorporation of new basis states into $\mathcal{H}_{M}$ when the model space is deemed adequate, with an error estimate 
below some predefined threshold. If the final estimate of the error is small, then the computed eigenstates and eigenenergies are still expected to be close to those obtained with full configuration interaction. In this case, since the latter method is size extensive (i.e., the computed energies scale properly with the number of particles [65]), this in practice permits a direct comparison between states of contrasting particle numbers even though ITCI is strictly speaking not size extensive by construction. In this work, the presented eigenenergies retrieved with ITCI (see Sec. IV) all have relative error estimates $\left|\delta E^{(2)} / E\right|<2.5 \times 10^{-5}$, if not explicitly stated otherwise. Still, in general, the rapid increase in the size of the Hilbert space $\mathcal{H}$ with $N$ effectively puts a limit to the applicability of the considered method. Even though not all Fock states belong to $\mathcal{H}_{M}$ (in which the diagonalization takes place), the importance of all (or at least a substantial part of) the Fock states in $\mathcal{H}_{C}$ also needs to be assessed in each iteration. With 702 one-body basis states, there are for instance already $\sim 1.00 \times 10^{10}$ possible many-body states to consider for $N=4$ (even though not all of them have the desired parity). A full configuration interaction calculation is thus here not feasible in practice. With the method of ITCI, on the other hand, after only a handful of iterations (starting with $\mathcal{H}_{M}$ spanned by the $\sim 10^{3}$ lowest-energy Fock states) the dimensionality of $\mathcal{H}_{M}$ will, in this case, typically have grown to $\sim 5 \times 10^{5}$ (which is substantially lower than that of $\mathcal{H}$ ) with errors of the individual eigenenergies decreased below the chosen threshold $\left|\delta E^{(2)} / E\right|=2.5 \times 10^{-5}$.

For slightly larger particle numbers (here for $N \leqslant 16$ ), we instead make use of the method of quadratic configuration interaction with single and double substitutions, QCI-SD. Here, the mean-field (Hartree-Fock) orbitals are first retrieved in the $\varphi$ basis given by Eq. (5). Next, to go beyond the mean-field descriptions, we compute (in a self-consistent procedure) the contributions connected to the many-body states constructed from the mean-field solution by single and double excitations, while at the same time enforcing size extensivity. The considered method can be seen as a modification of the configuration interaction with single and double substitutions, making it size extensive by adding appropriate terms in the projection equations [60]. Alternatively, QCI-SD may be thought of as a simplified version of the corresponding coupledcluster method with single and double substitutions. With QCI-SD and given the considered one-body basis size, only $\sim 1.46 \times 10^{6}$ many-body basis-states are, e.g., referenced for $N=4$ and $\sim 2.82 \times 10^{7}$ states for $N=16$. To speed up (and in some cases to facilitate) the convergence of the self-consistent QCI-SD calculation, the frequently used direct inversion of the iterative subspace (DIIS) method [66] is here implemented. Note also that, in order to construct the many-body low-energy spectrum, in addition QCI-SD is here applied to the excited Hartree-Fock solutions, converging to local minima in the energy surface. Even when only the many-body ground state is desired, for systems where the many-body ground and first excited states are close to one another in energy, we stress the necessity of also considering the excited Hartree-Fock solutions. When correcting for the correlation energy, an excited mean-field state may produce the lowest energy; i.e., an excited Hartree-Fock solution could be the mean-field approximation of the actual many-body ground state. To find the excited mean-field solutions, we here start the self-consistent iteration from different excitations of the mean-field ground state and use the method of maximum overlap [67] together with DIIS to support the desired convergences. Alternatively, in some cases, we instead map out the different mean-field (excited) solutions by following them individually during a numerical sweep in the dipolar orientation $\Theta$. A converged mean-field solution at one angle, $\Theta$, is thus used as the initial guess for the subsequent solution at a slightly larger, or smaller, angle.

Finally, we demonstrate that the choice of 702 singleparticle states of the form given in Eq. (5), with $26 B$-splines for the radial description and $m \in[-13,13]$ for the angular one, is sufficient for an adequate description of the considered systems (where $\alpha=1.15, D^{2}=0.8^{2} \hbar \omega_{\perp} l_{\perp}^{3}, N \leqslant 16$, and $45^{\circ} \leqslant \Theta \leqslant 90^{\circ}$ ). In particular, we increase the number of $B$-splines from 26 to 39 and investigate the effects on the numerically retrieved many-body ground-state energies using QCI-SD for $N=16$, which is the computationally most demanding case considered. Both the energy convergence with respect to an increase in the computational box radius and a decrease in the knot-point separation $\Delta \tau$ (enabling solutions with higher frequency modulations) are studied numerically. In the former case, we find that the many-body ground-state energy retrieved for $\Theta=90^{\circ}$ shifts from $\sim 79.770 \hbar \omega_{\perp}$ to $\sim 79.758 \hbar \omega_{\perp}$ when extending the computational box radius from $r_{\perp}=5 l_{\perp}$ (using $26 B$-splines) to $r_{\perp}=7.5 l_{\perp}$ (using 39 $B$-splines), while keeping $\Delta \tau=0.15$. We recall that, as discussed already in connection to Eqs. (6) and (7), the largest computational box radius is generally required for, and thus the largest energy shift (or error) will here be observed for, isotopic dipole-dipole interactions, i.e., for $\Theta=90^{\circ}$. Similarly, for $\Theta=45^{\circ}$ (where we expect the highest density of knot points to be needed), the energy shifts from $\sim 65.557 \hbar \omega_{\perp}$ to $\sim 65.524 \hbar \omega_{\perp}$ when reducing $\Delta \tau$ from $\Delta \tau=0.15$ (using $26 B$-splines) to $\Delta \tau=0.1$ (using $39 B$-splines) while keeping the box radius fixed at $5 l_{\perp}$. The rather small energy shifts encountered in both scenarios indicate that the considered basis of 702 single-particle states successfully captures the physical features of systems where $45^{\circ} \leqslant \Theta \leqslant 90^{\circ}$ and $N \leqslant 16$.

\section{LOW-ENERGY EIGENSTATES}

Let us now first examine the properties of the ground and the first excited state in the case of a few dipolar fermions, with interaction strength $D^{2}=0.8^{2} \hbar \omega_{\perp} l_{\perp}^{3}$, confined in a slightly anisotropic trap that is elongated in the $y$ direction ( $\alpha=1.15$ ). For $N=1$, the ground- and the first-excited-state energies are $E_{0} /\left(\hbar \omega_{\perp}\right) \approx 1.0024$ and $E_{1} /\left(\hbar \omega_{\perp}\right) \approx 1.9349$, respectively. Recalling the corresponding energies $E_{0} /\left(\hbar \omega_{\perp}\right)=$ 1 and $E_{1} /\left(\hbar \omega_{\perp}\right)=2$ for an isotropic harmonic trap, we here note that the anisotropic deformation shifts the two singleparticle energies in opposite directions, bringing them closer to one another. For larger particle numbers, the energy structure becomes more complex (owing to the interaction between the particles) and depends explicitly on the dipolar orientation angle $\Theta$ [61]. In the left panels of Fig. 2, we show the low many-body energies retrieved with the ITCI method, described in Sec. III above, for $N=2,3$, and 4, and a trap deformation of strength $\alpha=1.15$. The right panels show the 


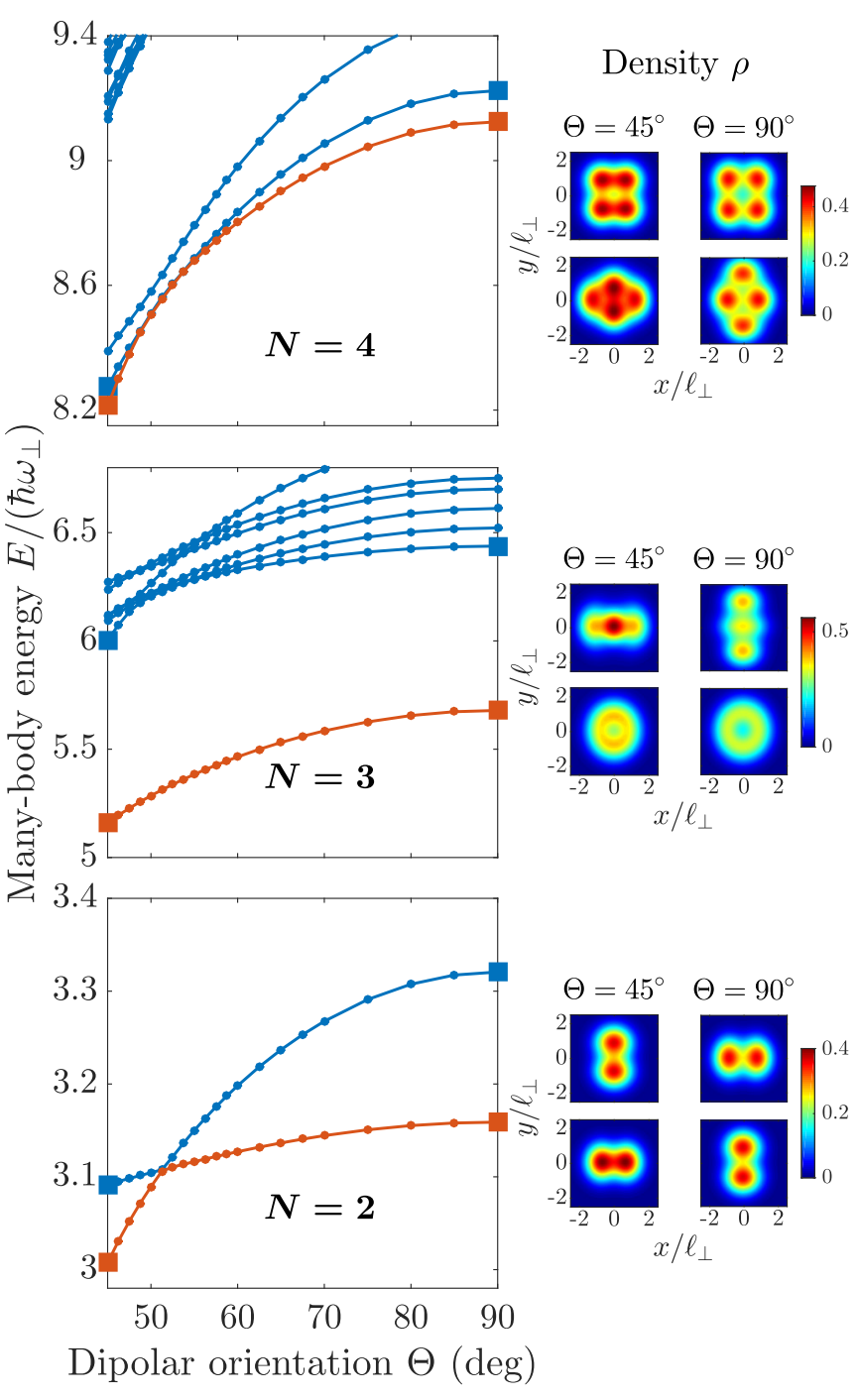

FIG. 2. The many-body energy associated with the ground state (red line) and the excited states (blue lines) for $2 \leqslant N \leqslant 4$ and for $45^{\circ} \leqslant \Theta \leqslant 90^{\circ}$. The panels to the right show the single-particle densities of the ground (last row) and first excited (first row) states at $\Theta=45^{\circ}$ (left column) and $\Theta=90^{\circ}$ (right column), for $\left|x / l_{\perp}\right| \leqslant 2.5$ and $\left|y / l_{\perp}\right| \leqslant 2.5$. The densities are normalized to $N$.

density distributions of the two-dimensional single-particle densities $\rho(x, y)$ for the many-body ground state and the first excited state at $\Theta=45^{\circ}$ and $\Theta=90^{\circ}$, corresponding to the larger squares seen in the left panels of the figure. For $\Theta=$ $90^{\circ}$, the isotropic repulsive dipole-dipole interaction results in ground-state densities that are shaped by the trap geometry. The larger extension of $\rho$ in the $y$ direction (when $\Theta=90^{\circ}$ ) is clearly seen for $N=2$ and $N=4$, but also holds for $N=3$. Upon reduction of $\Theta$, the anisotropy of the interaction potential increases. In particular, the effective dipole-dipole interaction becomes attractive for configurations where the particles are oriented "head-to-tail," i.e., for particles lined up in the $x$ direction. With the decrease in $\Theta$ follows thus a possible decrease of the effective interaction energy, and consequently of the energy of the many-body ground and lowlying states. Indeed, for $\Theta=45^{\circ}$ and for $N=2$ and $N=4$, we see that the ground-state densities are elongated in the $x$ direction instead, favoring head-to-tail configurations. The drastic change in ground-state density profiles are attributed to the (avoided) crossings seen around $\Theta \approx 50^{\circ}$ for $N=2$ and $N=4$. Actually, for $N=4$, there are three states of the same (even) parity and close in energy in the vicinity of $\Theta \approx 50^{\circ}$, which explains the different structure in $\rho$ between the first excited state at $\Theta=90^{\circ}$ and the ground state at $\Theta=45^{\circ}$. The higher-energy states for $N=4$ seen in the top left corner in the top panel, with $E /\left(\hbar \omega_{\perp}\right)>9$ for $\Theta=45^{\circ}$, all have odd parity and error estimates $\left|\delta E^{(2)} / E\right|<6 \times 10^{-5}$, in contrast to that of all other shown states where $\left|\delta E^{(2)} / E\right|<$ $2.5 \times 10^{-5}$. For $N=3$, the corresponding lowest excitation energy is larger and the change in the ground-state density distribution with $\Theta$ is less pronounced. However, the density profile of the first excited state changes greatly. Once again, (avoided) crossings are responsible for this particular change in $\rho$, making the density of the first excited state elongated in the $x$ direction at lower $\Theta$. Intriguingly, for the ground state, a similar decrease in $\Theta$ triggers a fundamentally different response in $\rho$. First of all, a closer look at the ground-state density reveals a minor increase in $\rho$ at the center of the trap for $\Theta=45^{\circ}$ compared to that for $\Theta=90^{\circ}$. In addition, a slightly lower value of $\left\langle x^{2}-y^{2}\right\rangle /$ $\left\langle x^{2}+y^{2}\right\rangle$ is found numerically for $\Theta=45^{\circ}$ compared to $\Theta=90^{\circ}$, where $\left\langle x^{2} \pm y^{2}\right\rangle=\iint\left(x^{2} \pm y^{2}\right) \rho(x, y) d x d y$. Even though it is hardly visible in Fig. 2, a decrease from $\Theta=90^{\circ}$ to $\Theta=45^{\circ}$ thus effectively increases the relative elongation of the ground-state density in the $y$ direction, i.e., in the orthogonal direction to what is favored by the anisotropic dipole-dipole interaction.

Let us now increase the number of fermions, $N$, to investigate if the system responds in a similar manner to a change in $\Theta$. In Fig. 3, the lowest excitation energies, $E_{1}-E_{0}$, retrieved with the method of QCI-SD (as discussed in Sec. III), are shown for $N \leqslant 10$. In general, we observe two different categories of many-body systems; those with large excitation energies (similar for $N=3,6$, and 10), and those with lower energy differences (similar for $N=2,4,5,7,8$, and 9). We also note that the latter systems all have minima in the excitation energy $E_{1}-E_{0}$ located somewhere in the region $50^{\circ} \lesssim \Theta \lesssim 55^{\circ}$. The inset in Fig. 3 shows the relative difference between the ground-state energies for $N \leqslant 4$ computed with ITCI (which is considered to be close to numerically exact) and QCI-SD. For $N=2$, QCI-SD will, by construction, replicate that of full configuration interaction. Clearly, the low deviations between the two methods also when $N=3$ and $N=4$ justifies the usage of QCI-SD (at least for low enough particle numbers).

We recall that the many-body ground state is characterized by the intricate interplay between the minimization of the (dipole-dipole) interaction energy and that of the single-particle energy dictated by the trapping potential. Furthermore, for certain $N$, an (avoided) energy crossing marks the transition from the single-particle density elongated in the $y$ direction (as favored by the trap) to one elongated in the $x$ direction (as favored by the dipole-dipole interaction when $\Theta<90^{\circ}$ ). For a larger interaction strength $D^{2}$ (not shown here), the crossing shifts closer to $\Theta=90^{\circ}$; i.e., a stronger contribution from the dipole-dipole interaction causes the system to respond already at smaller differences of the tilt 


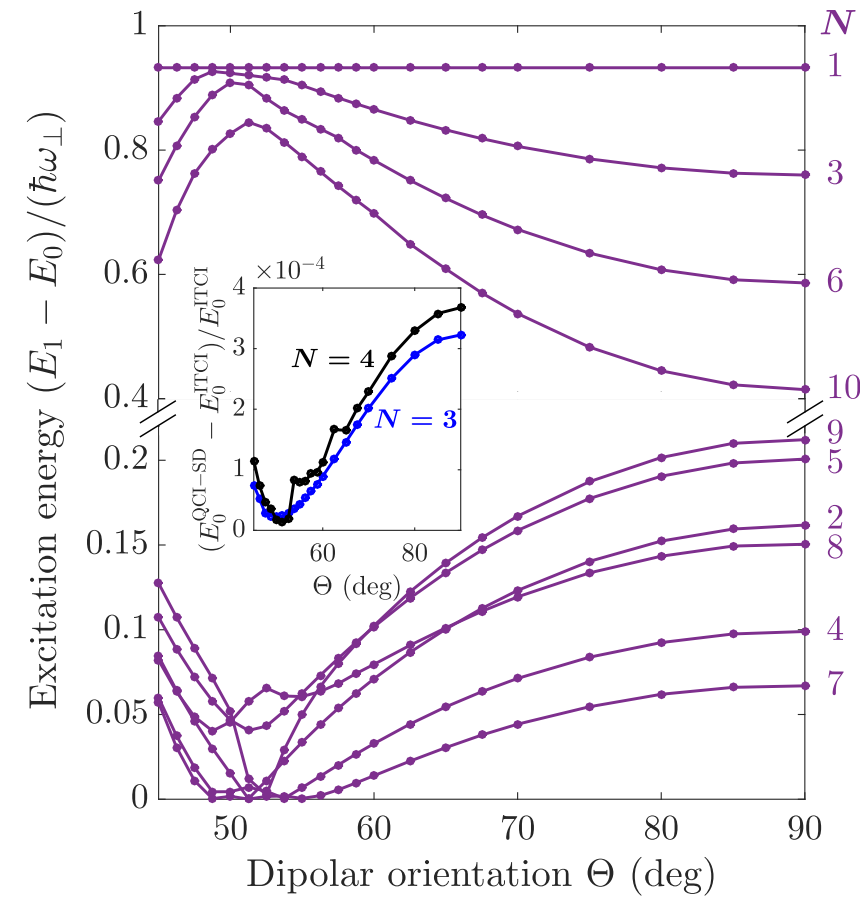

FIG. 3. The energy difference $E_{1}-E_{0}$, between the first excited and the ground state of the system, for $N \leqslant 10$. (Note the change of scale for the energies in the upper and lower part of the figure). For the given values of the dipolar orientation $\Theta$, the gaps are largest in the closed-shell configurations with $N=1,3,6$, and 10 . The inset shows a comparison between the ground-state energies computed with ITCI and QCI-SD for $N=3$ and $N=4$.

angle from the isotropic case $\Theta=90^{\circ}$. Similarly, a larger trap deformation $(\alpha>1.15)$ shifts the crossing further away from the value $\Theta=90^{\circ}$. Naturally, for $\alpha<1$ both the trap deformation and the tilt of the dipolar orientation (occurring in the $x z$ plane) favors systems elongated in the $x$ direction. The two effects thus enhance, rather than counteract, one another and no similar crossing, i.e., marking the transition between ground-state densities elongated in different directions, is expected.

\section{ENERGY SHELL STRUCTURE}

For comparison we now first briefly discuss the trivial case of noninteracting fermions in an isotropic harmonic confinement, i.e., a system where $D^{2}=0$ and $\alpha=1$. In this case, the system displays a pronounced energy shell structure, trivially reflecting the azimuthal symmetry of the one-body problem that leads to the degeneracies $d_{N_{0}}=N_{0}+1$ with shell index $N_{0}=0,1,2, \ldots$ The $N$-body ground state adheres to the aufbau principle, with fermions occupying the $N$ singleparticle states of lowest energy. For spin-polarized fermions, closed energy shell systems are consequently found for $N=$ $1,3,6,10,15, \ldots$ in agreement with the single-particle orbital degeneracy and with the Pauli exclusion principle.

Weakly interacting trapped fermions may exhibit energy shells reminiscent of those of the noninteracting particles if the states are not too strongly correlated, which has been observed for example also in quasi-two-dimensional and azimuthally symmetric quantum dots (see the review in Ref. [4]). For dipolar fermions in an isotropic harmonic trap, similar energy shells are thus to be expected in the purely repulsive case at $\Theta=90^{\circ}$ where the isotropic repulsion between particles renders the many-body problem rotationally invariant about the $z$ axis. The particle interaction, however, makes the description of distinct energy shells an approximate one, applicable only in a mean-field picture: The single-particle mean-field energies, although not necessarily being degenerate, may cluster around certain energy values, i.e., in different energy shells, with larger energy gaps in between them. For a fixed confinement, the interaction energy contribution to the many-body energy grows with $N$, typically reducing the resemblance between the interacting and the noninteracting systems.

Let us now return to the case of dipolar fermions with interaction strength $D^{2}=0.8^{2} \hbar \omega_{\perp} l_{\perp}^{3}$ in an anisotropic trap with $\alpha=1.15$. As a consequence of the trap deformation, the energy shell structure for $\Theta=90^{\circ}$ will (at least partly) be destroyed. As the single-particle orbital degeneracies associated with the isotropic harmonic oscillator are lifted, the energy shell structure is distorted already for noninteracting fermions. A reduction in $\Theta$ renders the effective dipole-dipole interaction anisotropic; i.e., also the rotational symmetry of the two-body part of the Hamiltonian gets broken. In the special case of an isotropic harmonic trap $(\alpha=1)$, such symmetry breaking necessarily weakens the energy shell resemblance for the system. For the deformed trap, however, the effect of changing $\Theta$ is more complex. The anisotropic interaction potential $V_{\perp}^{\text {int }}$ can here, in principle, counteract the effects of the trap deformation and largely restore the energy shell structure. Intriguingly, this revival of shell structure originates from the elaborate interplay between one-body physics (governed by the anisotropic trap) and two-body physics (where the anisotropy is an intrinsic property of the dipolar interaction).

For systems exhibiting a clear energy shell structure, the many-body ground states are (nearly) degenerate for the openshell systems. In other words, the lowest excitation energies observed for the open-shell systems are significantly lower than the corresponding ones for the closed-shell systems. In Fig. 3, such a feature in $E_{1}-E_{0}$ is observed (particularly for $50^{\circ} \lesssim \Theta \lesssim 55^{\circ}$ ) which hints at closed energy shells for $N=1,3,6,10, \ldots$, similar to the case of noninteracting spinpolarized fermions in a two-dimensional isotropic harmonic confinement.

To characterize the shell structure in the different manybody systems characterized by the particle number $N$, we compute the addition energy difference $[68,69]$

$$
\Delta_{2}(N)=\left[E_{0}(N+1)-E_{0}(N)\right]-\left[E_{0}(N)-E_{0}(N-1)\right],
$$

where $E_{0}(N)$ is the energy of the $N$-fermion ground state. When the shell structure is strong, one expects a series of pronounced peaks in $\Delta_{2}$ at the shell fillings which for a system close to a two-dimensional isotropic harmonic oscillator should occur at $N=1,3,6,10,15, \ldots$, as we argued for above. Noninteracting fermions in an isotropic harmonic trap have $\Delta_{2}(N) /\left(\hbar \omega_{\perp}\right)=1$ for the closed-shell $N$-particle systems and $\Delta_{2}(N) /\left(\hbar \omega_{\perp}\right)=0$ for the open ones. In Fig. 4, we show the $\Delta_{2}$ obtained with the method of QCI-SD for 




FIG. 4. The difference in addition energies, $\Delta_{2}(N)$, for dipolar fermions in the anisotropic confinement $\alpha=1.15$. Two different dipolar orientations are considered: $\Theta=90^{\circ}$ (yellow line) and $\Theta=$ $52.5^{\circ}$ (red line) with interaction strength $D^{2}=0.8^{2} \hbar \omega_{\perp} l_{\perp}^{3}$. In addition, the case of noninteracting fermions $\left(D^{2}=0\right.$, black dotted line) is shown as a reference.

$D^{2}=0.8^{2} \hbar \omega_{\perp} l_{\perp}^{3}, N=1,2, \ldots, 15$, and for $\Theta=52.5^{\circ}(\mathrm{red}$ solid line), i.e., for the dipolar orientation where we expect energy shells to manifest. For comparison, we also include $\Delta_{2}$ for the case where $\Theta=90^{\circ}$ (yellow solid line) as well as for noninteracting fermions (black dotted line). Note that the latter two cases both show a rapid decline in the peak heights of $\Delta_{2}$ with $N$, indicating a loss of energy shell structure due to the deformation of the trap. For $\Theta=52.5^{\circ}$, on the other hand, a remarkable revival of the energy shell structure can be seen, also showing a smaller decrease in peak height in $\Delta_{2}$ with $N$. (We here recall that, for a fixed confinement, a zero decay rate is, in general, not possible due to the increased contribution from the interaction energy with $N$ ).

Finally, in Fig. 5, we examine the properties of $\Delta_{2}$ for different dipolar orientations in the moderately deformed trap with $\alpha=1.15$ in more detail. We find that the largest peak heights of $\Delta_{2}$ associated with the closed-shell systems are indeed obtained for $50^{\circ} \lesssim \Theta \lesssim 55^{\circ}$, i.e., the dipolar orientation giving rise to the lowest excitation energies in Fig. 3. We also note the slight shift in the peak heights in $\Delta_{2}$ (for $\left.N=3,6,10,15\right)$ towards higher $\Theta$ for larger $N$. A similar tendency can be seen in the corresponding maxima in the excitation energies in Fig. 3. We repeat, the larger number of particles increases the influence from the dipole-dipole interaction, which makes an angle closer to the isotropic $\Theta=90^{\circ}$ favorable for shell structures. In Fig. 5 we also observe that correlations enhance the peaks in $\Delta_{2}$ making the shell structure more pronounced. Next, we compare the shell structure retrieved for tilted dipoles, $\Theta=52.5^{\circ}$, in a deformed trap, $\alpha=1.15$ (middle panel), to the corresponding one for dipoles with $\Theta=90^{\circ}$ in an isotropic harmonic confinement where $\alpha=1.0$ (right panel). Clearly, for the isotropic trap, the peaks in $\Delta_{2}$ for closed shells are, at least for the first shells, higher than the corresponding ones for the deformed trap. On the other hand, the peak height in $\Delta_{2}$ decreases more rapidly with $N$ for the isotropic harmonic trap and drops already at $N=10$ below the corresponding one for $\alpha=1.15$ and $\Theta=52.5^{\circ}$. In other words, tilted dipoles in a

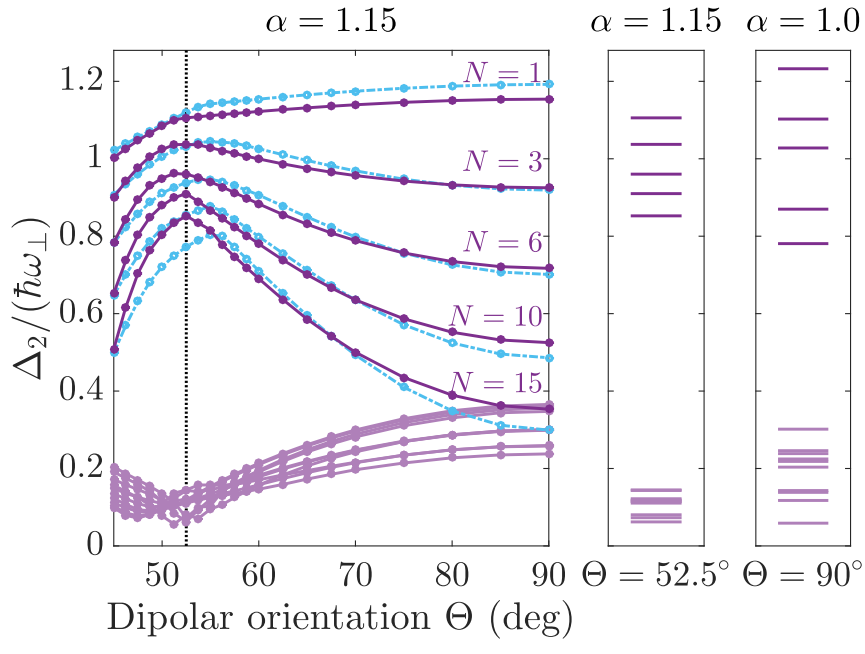

FIG. 5. Addition energy differences as a function of $\Theta$ for fermions in a deformed trap with $\alpha=1.15$ (left panel). The addition energy difference is shown for $\Theta=52.5^{\circ}$ (middle panel) where the shell structure is most pronounced (see the dashed vertical line in the left panel) and, as a reference, for fermions with fixed dipolar orientation, $\Theta=90^{\circ}$, in an isotropic harmonic confinement, $\alpha=1$ (right panel). In all three cases, the interaction strength is $D^{2}=0.8^{2} \hbar \omega_{\perp} l_{\perp}^{3}$. The purple solid lines are obtained using QCI-SD, whereas the blue dashed lines correspond to $\Delta_{2}$ computed directly from the HartreeFock energies for $N=1,3,6,10,15$. The purple lines associated with closed-shell systems are labeled by their particle numbers $N$ for $\alpha=1.15$. Although not shown explicitly, in both panels to the right the descending order of $\Delta_{2}$ is also given by $N=1,3,6,10,15$.

deformed trap seem to extend the shell structure to slightly higher particle numbers. Here, the anisotropy of the tilted dipole-dipole interaction effectively reduces the interaction energy (by introducing attractive regions) and thus prolongs the energy shell structure of the system.

\section{CONCLUSIONS}

To summarize, we here investigated the effect of an anisotropic dipole-dipole interaction between (polarized) fermions on the shell structure in a quasi-two-dimensional harmonic oscillator confinement. The interplay between magnetostriction caused by a tilt in the dipolar angle [37], and the stability of shells following a break in the rotational symmetry triggered by a quadrupole deformation of the isotropic trap, was examined. Even though both considered processes lower the symmetry of the Hamiltonian in effectively similar ways, their nature is inherently different: A deformation of the trap alters directly the one-body part of the problem, whereas a change in the interaction instead modifies the two-body physics.

We found that, also for weakly deformed traps, the underlying shell structure of the isotropic harmonic oscillator persists for modest interaction strengths and tilt angles, at least for lower particle numbers (in the present work for $N \sim 10$ ). At open shells, however, there is generally a notable deformation of the density distribution, caused by the dipolar magnetostriction $[47,55]$. However, this deformation can be counteracted upon by the corresponding quadrupolar defor- 
mation of the isotropic trap and thus leading to a revival of energy shell structure resembling the azimuthally symmetric case of aligned dipoles perpendicular to the trap plane.

Giving an outlook, it would be interesting to investigate the modifications and breakdown of shell structure for stronger interactions, and especially to study the interplay with the two-component properties of isospin in mixtures, such as recently experimentally realized with erbium [36]. Especially in the mid-shell regime, where for weakly interacting gases Hund's rules prevail, tunable interspin interactions in combination with the dipolar anisotropy here offer new perspectives. Likewise, the extension to spin-orbit-coupled dipolar Fermi gases (see Ref. [70] and references therein) appears highly relevant. We finally note that it would be most interesting to

[1] W. A. de Heer, Rev. Mod. Phys. 65, 611 (1993).

[2] M. Brack, Rev. Mod. Phys. 65, 677 (1993).

[3] A. Bohr and B. R. Mottelson, Nuclear Structure: Volume I, Single-Particle Motion; Volume II, Nuclear Deformations (World Scientific, Singapore, 1998).

[4] S. M. Reimann and M. Manninen, Rev. Mod. Phys. 74, 1283 (2002).

[5] F. Dalfovo, S. Giorgini, L. P. Pitaevskii, and S. Stringari, Rev. Mod. Phys. 71, 463 (1999).

[6] C. J. Pethick and H. Smith, Bose-Einstein Condensation in Dilute Gases, 2nd ed. (Cambridge University Press, Cambridge, U.K., 2008).

[7] L. Pitaevskii and S. Stringari, Bose-Einstein Condensation, International Series of Monographs on Physics (Oxford University Press, New York, 2003).

[8] S. Giorgini, L. P. Pitaevskii, and S. Stringari, Rev. Mod. Phys. 80, 1215 (2008).

[9] I. Bloch, J. Dalibard, and W. Zwerger, Rev. Mod. Phys. 80, 885 (2008).

[10] J. F. Sherson, C. Weitenberg, M. Endres, M. Cheneau, I. Bloch, and S. Kuhr, Nature 467, 68 (2010).

[11] W. S. Bakr, A. Peng, M. E. Tai, R. Ma, J. Simon, J. I. Gillen, S. Foelling, L. Pollet, and M. Greiner, Science 329, 547 (2010).

[12] C. Weitenberg, M. Endres, J. F. Sherson, M. Cheneau, P. Schauß, T. Fukuhara, I. Bloch, and S. Kuhr, Nature 471, 319 (2011).

[13] F. Serwane, G. Zürn, T. Lompe, T. B. Ottenstein, A. N. Wenz, and S. Jochim, Science 332, 336 (2011).

[14] S. Giovanazzi, A. Görlitz, and T. Pfau, Phys. Rev. Lett. 89, 130401 (2002).

[15] J. Werner, A. Griesmaier, S. Hensler, J. Stuhler, T. Pfau, A. Simoni, and E. Tiesinga, Phys. Rev. Lett. 94, 183201 (2005).

[16] A. Griesmaier, J. Werner, S. Hensler, J. Stuhler, and T. Pfau, Phys. Rev. Lett. 94, 160401 (2005).

[17] J. Stuhler, A. Griesmaier, T. Koch, M. Fattori, T. Pfau, S. Giovanazzi, P. Pedri, and L. Santos, Phys. Rev. Lett. 95, 150406 (2005).

[18] T. Koch, T. Lahaye, J. Metz, B. Fröhlich, A. Griesmaier, and T. Pfau, Nat. Phys. 4, 218 (2008). bring current experiments with dipolar Fermi gases into the few-body regime. For nondipolar fermions, new atom imaging schemes in two dimensions $[71,72]$ recently revealed a phase transition precursor. Their combination with long-ranged interactions may also open new avenues to explore the interplay between shell structure and interactions in low-dimensional fermion systems.

\section{ACKNOWLEDGMENTS}

We thank E. Ö. Karabulut, L. H. Kristinsdóttir, F. Malet Giralt, and M. Rontani for discussions at an early stage of this project. Our work was financially supported by the Swedish Research Council, the Knut and Alice Wallenberg Foundation, and NanoLund.
[19] T. Lahaye, C. Menotti, L. Santos, M. Lewenstein, and T. Pfau, Rep. Prog. Phys. 72, 126401 (2009).

[20] M. Lu, S. H. Youn, and B. L. Lev, Phys. Rev. Lett. 104, 063001 (2010).

[21] M. Lu, N. Q. Burdick, S. H. Youn, and B. L. Lev, Phys. Rev. Lett. 107, 190401 (2011).

[22] H. Kadau, M. Schmitt, M. Wenzel, C. Wink, T. Maier, I. FerrierBarbut, and T. Pfau, Nature 530, 194 (2016).

[23] K. Aikawa, A. Frisch, M. Mark, S. Baier, A. Rietzler, R. Grimm, and F. Ferlaino, Phys. Rev. Lett. 108, 210401 (2012).

[24] K.-K. Ni, S. Ospelkaus, M. De Miranda, A. Pe'Er, B. Neyenhuis, J. Zirbel, S. Kotochigova, P. Julienne, D. Jin, and J. Ye, Science 322, 231 (2008).

[25] K. K. Ni, S. Ospelkaus, D. Wang, G. Quéméner, B. Neyenhuis, M. H. G. de Miranda, J. L. Bohn, J. Ye, and D. S. Jin, Nature 464, 1324 (2010).

[26] M. H. G. de Miranda, A. Chotia, B. Neyenhuis, D. Wang, G. Quéméner, S. Ospelkaus, J. L. Bohn, J. Ye, and D. S. Jin, Nat. Phys. 7, 502 (2011).

[27] J. W. Park, S. A. Will, and M. W. Zwierlein, Phys. Rev. Lett. 114, 205302 (2015).

[28] S. A. Moses, J. P. Covey, M. T. Miecnikowski, B. Yan, B. Gadway, J. Ye, and D. S. Jin, Science 350, 659 (2015).

[29] A. Frisch, M. Mark, K. Aikawa, S. Baier, R. Grimm, A. Petrov, S. Kotochigova, G. Quéméner, M. Lepers, O. Dulieu, and F. Ferlaino, Phys. Rev. Lett. 115, 203201 (2015).

[30] L. De Marco, G. Valtolina, K. Matsuda, W. G. Tobias, J. P. Covey, and J. Ye, Science 363, 853 (2019).

[31] M. Lu, N. Q. Burdick, and B. L. Lev, Phys. Rev. Lett. 108, 215301 (2012).

[32] K. Aikawa, A. Frisch, M. Mark, S. Baier, R. Grimm, and F. Ferlaino, Phys. Rev. Lett. 112, 010404 (2014).

[33] B. Naylor, A. Reigue, E. Maréchal, O. Gorceix, B. LaburtheTolra, and L. Vernac, Phys. Rev. A 91, 011603(R) (2015).

[34] M. Baranov, Phys. Rep. 464, 71 (2008).

[35] M. A. Baranov, M. Dalmonte, G. Pupillo, and P. Zoller, Chem. Rev. 112, 5012 (2012).

[36] S. Baier, D. Petter, J. H. Becher, A. Patscheider, G. Natale, L. Chomaz, M. J. Mark, and F. Ferlaino, Phys. Rev. Lett. 121, 093602 (2018). 
[37] T. Miyakawa, T. Sogo, and H. Pu, Phys. Rev. A 77, 061603(R) (2008).

[38] J.-N. Zhang and S. Yi, Phys. Rev. A 80, 053614 (2009).

[39] D. Baillie and P. B. Blakie, Phys. Rev. A 82, 033605 (2010).

[40] J.-N. Zhang and S. Yi, Phys. Rev. A 81, 033617 (2010).

[41] A. R. P. Lima and A. Pelster, Phys. Rev. A 81, 063629 (2010).

[42] A. R. P. Lima and A. Pelster, Phys. Rev. A 81, 021606(R) (2010).

[43] S. Ronen and J. L. Bohn, Phys. Rev. A 81, 033601 (2010).

[44] B. Liu and L. Yin, Phys. Rev. A 84, 053603 (2011).

[45] D. Baillie and P. B. Blakie, Phys. Rev. A 86, 023605 (2012).

[46] D. Baillie, R. N. Bisset, and P. B. Blakie, Phys. Rev. A 91, 013613 (2015)

[47] V. Veljić, A. Balaž, and A. Pelster, Phys. Rev. A 95, 053635 (2017).

[48] V. Veljić, A. R. P. Lima, L. Chomaz, S. Baier, M. J. Mark, F. Ferlaino, A. Pelster, and A. Balaž, New J. Phys. 20, 093016 (2018).

[49] K. Góral, B.-G. Englert, and K. Rzążewski, Phys. Rev. A 63, 033606 (2001).

[50] K. Aikawa, S. Baier, A. Frisch, M. Mark, C. Ravensbergen, and F. Ferlaino, Science 345, 1484 (2014).

[51] J. Schneider and H. Wallis, Phys. Rev. A 57, 1253 (1998).

[52] P. Vignolo and A. Minguzzi, Phys. Rev. A 67, 053601 (2003).

[53] Y. Yu, M. Ögren, S. Åberg, S. M. Reimann, and M. Brack, Phys. Rev. A 72, 051602(R) (2005).

[54] M. Tohyama, J. Phys. Soc. Jpn. 78, 104003 (2009).

[55] V. Veljic, A. Pelster, and A. Balaž, Phys. Rev. Res. 1, 012009 (2019).
[56] M. M. Parish and F. M. Marchetti, Phys. Rev. Lett. 108, 145304 (2012).

[57] H. Ustunel, S. H. Abedinpour, and B. Tanatar, J. Phys.: Conf. Ser. 568, 012020 (2014).

[58] F. Ancilotto, Phys. Rev. A 92, 061602(R) (2015).

[59] R. Roth, Phys. Rev. C 79, 064324 (2009).

[60] J. A. Pople, M. Head-Gordon, and K. Raghavachari, J. Chem. Phys. 87, 5968 (1987).

[61] J. C. Cremon, G. M. Bruun, and S. M. Reimann, Phys. Rev. Lett. 105, 255301 (2010).

[62] C. De Boor, A Practical Guide to Splines, Applied Mathematical Sciences Vol. 27 (Springer, New York, 1978)

[63] P. S. Epstein, Phys. Rev. 28, 695 (1926).

[64] R. K. Nesbet and D. R. Hartree, Proc. R. Soc. London, Ser. A 230, 312 (1955).

[65] R. J. Bartlett, Annu. Rev. Phys. Chem. 32, 359 (1981).

[66] P. Pulay, Chem. Phys. Lett. 73, 393 (1980).

[67] A. T. B. Gilbert, N. A. Besley, and P. M. W. Gill, J. Phys. Chem. A 112, 13164 (2008).

[68] K. Capelle, M. Borgh, K. Kärkkäinen, and S. M. Reimann, Phys. Rev. Lett. 99, 010402 (2007).

[69] M. Rontani, J. R. Armstrong, Y. Yu, S. Åberg, and S. M. Reimann, Phys. Rev. Lett. 102, 060401 (2009).

[70] N. Q. Burdick, Y. Tang, and B. L. Lev, Phys. Rev. X 6, 031022 (2016).

[71] M. Holten, L. Bayha, K. Subramanian, C. Heintze, P. M. Preiss, and S. Jochim, arXiv:2005.03929.

[72] L. Bayha, M. Holten, R. Klemt, K. Subramanian, J. Bjerlin, S. M. Reimann, G. M. Bruun, P. M. Preiss, and S. Jochim, arXiv:2004.14761. 